\title{
Overnight T-Wave Alternans in Sleep Apnea Patients
}

\author{
Laura Burattini, Ilaria Ciotti, Michela D'Ignazio, Alessandro Miccoli, Angela Agostinelli, \\ Agnese Sbrollini, Micaela Morettini, Francesco Di Nardo, Sandro Fioretti
}

Università Politecnica delle Marche, Ancona, Italy

\begin{abstract}
Sleep apnea (SA) is linked to cardiovascular complications and to an increased risk of sudden cardiac death. Microvolt T-wave alternans (TWA) is a noninvasive electrocardiographic (ECG) index of cardiovascular risk; its rate of occurrence in SA patients remains unknown. Thus, this study investigated the occurrence of TWA in SA patients during night. To this aim, overnight ECG recordings of 16 SA patients were analyzed for TWA identification by means of our heart rate adaptive match filter. Results indicate that overnight TWA was characterized by a low mean amplitude (mean TWA: $6 \pm 3 \mu V)$. However, higher-amplitude transient TWA episodes (max TWA: $29 \pm 21 \mu \mathrm{V}$ ) occurred overnight, sometimes when patients were awake (max TWA: $33 \pm 18$ $\mu \mathrm{V} ; 56 \%$ of cases) and sometimes when patients were sleeping (max TWA: $24 \pm 23 \mu \mathrm{V}$; $44 \%$ of cases with $13 \%$, $19 \%, 6 \%$ and $6 \%$ during sleep stage 1, 2, 3 and 4, respectively). In only 3 subjects (19\%) TWA peaks occurred during an SA episode: during obstructive apnea with arousal in two cases (max TWA of $7 \mu \mathrm{V}$ and $17 \mu \mathrm{V}$, during stages 1 and 2 , respectively) and during hypoapnea with arousal in one case (max TWA of $6 \mu \mathrm{V}$ while awake). Thus, SA patients show significant transient overnight TWA episodes, not necessarily occurring during a SA episode.
\end{abstract}

\section{Introduction}

Sleep is a periodic state of mind and body that differs from wakefulness because of a decreased ability to react to stimuli, a reduced consciousness, an almost complete inhibition of sensory and voluntary-muscles activities, and a drastic reduction of interactions with surroundings. By analyzing an electroencephalogram, sleep can be divided into two phases: non rapid eye movement (nonREM), also known as quiet sleep, and REM, also known as active sleep or paradoxical sleep. The non-REM phase can be further classified in stages 1 to 4 . Stage 1 corresponds to drowsiness: eyes move slowly and muscle activity decreases. Stage 2 corresponds to light sleep: eyes do not move and heart rate (HR) and breathing decrease. Stages 3 and 4 correspond to deep sleep: no eye movement and no muscle activity occur. Eventually, during the REM phase, rapid eye movements occur and HR and breathing increase. Sleep does not sequentially progress through the five stages and cycles every 90-120 minutes through them approximately four or five times per night.

Good sleep is necessary for optimal health and sleep disorders may affect hormone levels, mood and weight. Sleep apnea (SA) is one of the most common sleep disorders. It affects about $10 \%$ of adults in the general population and may overcome 50\% in certain cardiovascular diseases involving sodium and water retention [1-4]. SA is a disorder in which breathing pauses of at least 10-15 s occur while sleeping. Breathing pauses can last as long as minutes and may reach an occurrence frequency of more than 30 times per hour. It can be classified in obstructive apnea (OA), the most common, when due to a collapse of the upper airway, or central apnea (CA), when due to a loss of ventilatory drive [2]. Hypopnea (HA) is less severe than SA and occurs when a shallow and slow breathing reduces the oxygen supply to lungs.

Although not yet used for cardiovascular risk assessment, there is a growing evidence that SA may cause or promote several cardiovascular diseases among which hypertension, coronary artery disease, heart failure and arrhythmias, and thus may be used as an index of risk for cardiovascular death $[1,5,6,7,8]$. Microvolt T-wave alternans (TWA), consisting in a subtle alternation of the electrocardiographic (ECG) $\mathrm{T}$ wave, is a promising noninvasive indicator of sudden cardiac death [9]. Although low-levels of TWA have been also observed in resting healthy subjects $[10,11,12]$, TWA is more commonly found in patients affected by cardiovascular diseases $[11,13,14,15,16]$ and its amplitude tends to increase with increasing heart rate (HR) $[9,17,18]$. As a consequence, since HR decrease during sleep and TWA is more hardly detectable, only few studies have investigated TWA incidence during sleep, especially when SA occurs $[7,19]$. Thus, aim of this study was to investigate the occurrence of TWA in SA patients during night to evaluate if TWA represents a potential tool to identify SA subjects at increased cardiovascular risk. 


\section{Methods}

\subsection{Clinical data}

Clinical data consisted of sixteen 2h-to-7h long polysomnographic recordings all including several physiological signals (sampling rate: $250 \mathrm{~Hz}$; resolution: 12 bit), among which a respiratory signal, a single channel ECG signal, an electroencephalographic signal, and an invasive blood pressure signal. Sleep stages and apnea were annotated every $30 \mathrm{~s}$ according to the Rechtschaffen and Kales's criteria [20]. Subjects were monitored in Boston's Beth Israel Hospital Sleep Laboratory for evaluation of chronic obstructive sleep apnea syndrome and to test the effects of continuous positive airway pressure, a standard intervention that generally prevents or reduce airway obstruction in pathological conditions. Data are available in the MITBIH Polysomnographic Database [21] by PhysioNet [22].

\subsection{Automatic T-wave alternans identification}

Automatic TWA identification was performed in four steps: windowing, preprocessing, suitability testing and adaptive filtering [23,24].

Windowing. From each recording, ECG windows containing 128 consecutive heart beats were recursively extracted with step of $30 \mathrm{~s}$ in order to match respiration windows used to annotate apnea.

Preprocessing. Each extracted ECG window was initially cleaned using a band-pass bidirectional $6^{\text {th }}$-order Butterworth filter (cut-off frequencies: $0.5 \mathrm{~Hz}$ and $45 \mathrm{~Hz}$ ). Then, $\mathrm{R}$ peaks were detected and used to compute mean RR interval (RR), HR (as 1/RR), and RR-interval standard deviation (RRSD) over the window. Baseline was estimated by a $3^{\text {rd }}$ order spline interpolation of fiducial points located $70 \mathrm{~ms}$ before the $\mathrm{R}$ peaks and subtracted from the ECG window. Ectopic and corrupted beats were replaced by the median beat.

Suitability test. Once preprocessed, the ECG window was tested to evaluate its suitability for TWA identification. Specifically, if $R R S D \leq 0.1 \cdot R R$ and there were at most 10 replaced beats the window was considered suitable, otherwise it was rejected (i.e. considered not suitable for TWA identification).

Heart-rate adaptive match filter. TWA was automatically detected and quantified within each window using the heart-rate adaptive match filter (HRAMF) [23,24]. After having computed the TWA fundamental frequency (FTWA) as $1 /(2 \cdot \mathrm{RR})$, HRAMF used FTWA to adaptively design a narrowband-pass filter with a $0.12 \mathrm{~Hz}$ wide passing band centred in FTWA. HRAMF was implemented as a $6^{\text {th }}$-order bidirectional Butterworth band-pass filter. After having processed the
ECG window, HRAMF provided a pseudo-sinusoidal signal that, in case of TWA, had maxima and minima occurring in correspondence of the ST segment or T wave. TWA amplitude was directly given by the pseudosinusoid amplitude.

\subsection{Statistics}

Each recording was characterized in terms of: mean HR, expressed as mean RR (MRR); HR variability (HRV), quantified as RR standard deviation; mean TWA; TWA variability (STD TWA), quantified as TWA standard deviation; and maximum TWA (max TWA). All statistics were quantified over the entire duration of the recording. Overnight association between HR and HRV and TWA was evaluated by computation of the correlation coefficient $(\rho)$. Statistical significance $(P)$ was set at 0.05 .

\section{Results}

An example of overnight TWA and RR trends are represented in Fig. 1 that shows how TWA was generally low amplitude but, occasionally, it may show transient higher-amplitude episodes, usually close to regions with too high RRSD (dotted lines) for TWA to be detected.

Overall, overnight TWA was characterized by a low mean amplitude (mean TWA: $6 \pm 3 \mu \mathrm{V}$; Table 1) and was associated to a quite low mean HR $(71 \pm 8 \mathrm{bpm}$ corresponding to MRR $=851 \pm 85 \mathrm{~ms}$; Table 1 ). However, higher-amplitude transient TWA episodes (max TWA: $29 \pm 21 \mu \mathrm{V}$; Table 1) occurred overnight, sometimes when patients were awake (max TWA: $33 \pm 18 \mu \mathrm{V} ; 56 \%$ of cases) and sometimes when patients were non-REM sleeping (max TWA: $24 \pm 23 \mu \mathrm{V} ; 44 \%$ of cases with $13 \%$, $19 \%, 6 \%$ and $6 \%$ during sleep stage 1,2, 3 and 4 , respectively; Table 1). No high amplitude TWA occurred during REM sleeping. Within the accepted HRV levels, TWA peaks increased with $\operatorname{HRV}(\rho=0.50, \mathrm{P}<0.05)$. In only 3 subjects (19\%) max TWA occurred during an apnea episode: during obstructive apnea with arousal in two cases (max TWA of $7 \mu \mathrm{V}$ and $17 \mu \mathrm{V}$, during stages 1 and 2, respectively; Table 1) and during hypopnea with arousal in one case (max TWA of $6 \mu \mathrm{V}$ while awake; Table 1).

\section{Discussion}

This study investigated the occurrence of TWA in SA during night. Overnight TWA was automatically identified by using the HRAMF, a method particularly suitable for TWA identification is case of low HR (such as during sleeping) and relatively high $\operatorname{HRV}[10,23]$. Still, in our SA patients, on average 55\% of ECG windows were rejected because characterized by too high HRV 
Table 1. Overnight HR and TWA features

\begin{tabular}{|c|c|c|c|c|c|c|c|c|c|c|}
\hline Sbj & $\begin{array}{l}\text { Overnight } \\
\text { apnea }\end{array}$ & $\begin{array}{c}\text { \#suitable } \\
\text { wnd }\end{array}$ & $\begin{array}{c}\text { MRR } \\
(\mathrm{ms})\end{array}$ & $\begin{array}{l}\text { HRV } \\
(\mathrm{ms})\end{array}$ & $\begin{array}{l}\text { mean } \\
\text { TWA } \\
(\mu \mathrm{V})\end{array}$ & $\begin{array}{l}\text { STD } \\
\text { TWA } \\
(\mu V)\end{array}$ & $\begin{array}{c}\max \\
\mathrm{TWA} \\
(\mu \mathrm{V})\end{array}$ & $\begin{array}{c}\text { RR } \\
\text { @ max } \\
\text { TWA } \\
(\mathrm{ms})\end{array}$ & $\begin{array}{l}\text { Sleep } \\
@ \text { max } \\
\text { TWA }\end{array}$ & $\begin{array}{l}\text { Apnea } \\
@ \text { max } \\
\text { TWA }\end{array}$ \\
\hline 1 & HA & $537(90 \%)$ & 948 & 53 & 6 & 5 & 56 & 950 & 4 & $*$ \\
\hline 2 & ,HA,OA & $294(47 \%)$ & 697 & 58 & 6 & 3 & 20 & 751 & 2 & - \\
\hline 3 & $\mathrm{HA}, \mathrm{OA}, \mathrm{CA}$ & $42(6 \%)$ & 878 & 30 & 5 & 1 & 8 & 836 & 3 & - \\
\hline 4 & $\mathrm{OA}$ & $254(35 \%)$ & 768 & 46 & 6 & 7 & 46 & 755 & W & - \\
\hline 5 & HA,OA & $453(63 \%)$ & 971 & 46 & 5 & 4 & 29 & 921 & W & - \\
\hline 6 & $\mathrm{HA}, \mathrm{OA}, \mathrm{CA}$ & $359(50 \%)$ & 801 & 77 & 14 & 6 & 43 & 846 & W & - \\
\hline 7 & $\mathrm{OA}$ & $188(30 \%)$ & 917 & 57 & 8 & 3 & 22 & 860 & W & - \\
\hline 8 & OA & $271(39 \%)$ & 669 & 27 & 6 & 2 & 17 & 635 & 2 & $\mathrm{OA}^{*}$ \\
\hline 9 & none & $470(61 \%)$ & 906 & 92 & 4 & 5 & 45 & 860 & W & - \\
\hline 10 & none & $633(84 \%)$ & 830 & 59 & 10 & 6 & 58 & 759 & 1 & - \\
\hline 11 & HA,OA & $334(44 \%)$ & 938 & 68 & 9 & 5 & 41 & 831 & $\mathrm{~W}$ & - \\
\hline 12 & $\mathrm{HA}, \mathrm{OA}, \mathrm{CA}$ & $285(60 \%)$ & 855 & 63 & 2 & 1 & 6 & 889 & $\mathrm{~W}$ & HA \\
\hline 13 & $\mathrm{HA}, \mathrm{OA}, \mathrm{CA}$ & $568(80 \%)$ & 845 & 54 & 3 & 1 & 7 & 944 & 1 & $\mathrm{OA}^{*}$ \\
\hline 14 & $\mathrm{HA}, \mathrm{OA}, \mathrm{CA}$ & $416(57 \%)$ & 902 & 66 & 5 & 5 & 59 & 809 & W & - \\
\hline 15 & $\mathrm{HA}, \mathrm{OA}$ & $244(56 \%)$ & 827 & 50 & 1 & 1 & 4 & 670 & W & - \\
\hline 16 & $\mathrm{OA}, \mathrm{CA}$ & $120(80 \%)$ & 866 & 35 & 2 & 1 & 4 & 875 & 2 & - \\
\hline tot & & $\begin{array}{c}342 \pm 163 \\
(55 \pm 22 \%)\end{array}$ & $851 \pm 85$ & $55 \pm 17$ & $6 \pm 3$ & $3 \pm 2$ & $29 \pm 21$ & $824 \pm 91$ & & \\
\hline
\end{tabular}

Sleep classification. 1-sleep stage 1; 2 sleep stage 2; 3: sleep stage 3; 4: sleep stage 4; W: awake.

Apnea classification. CA: central apnea; HA: hypopnea; OA: obstructive apnea with arousal; -: no apnea *: arousal

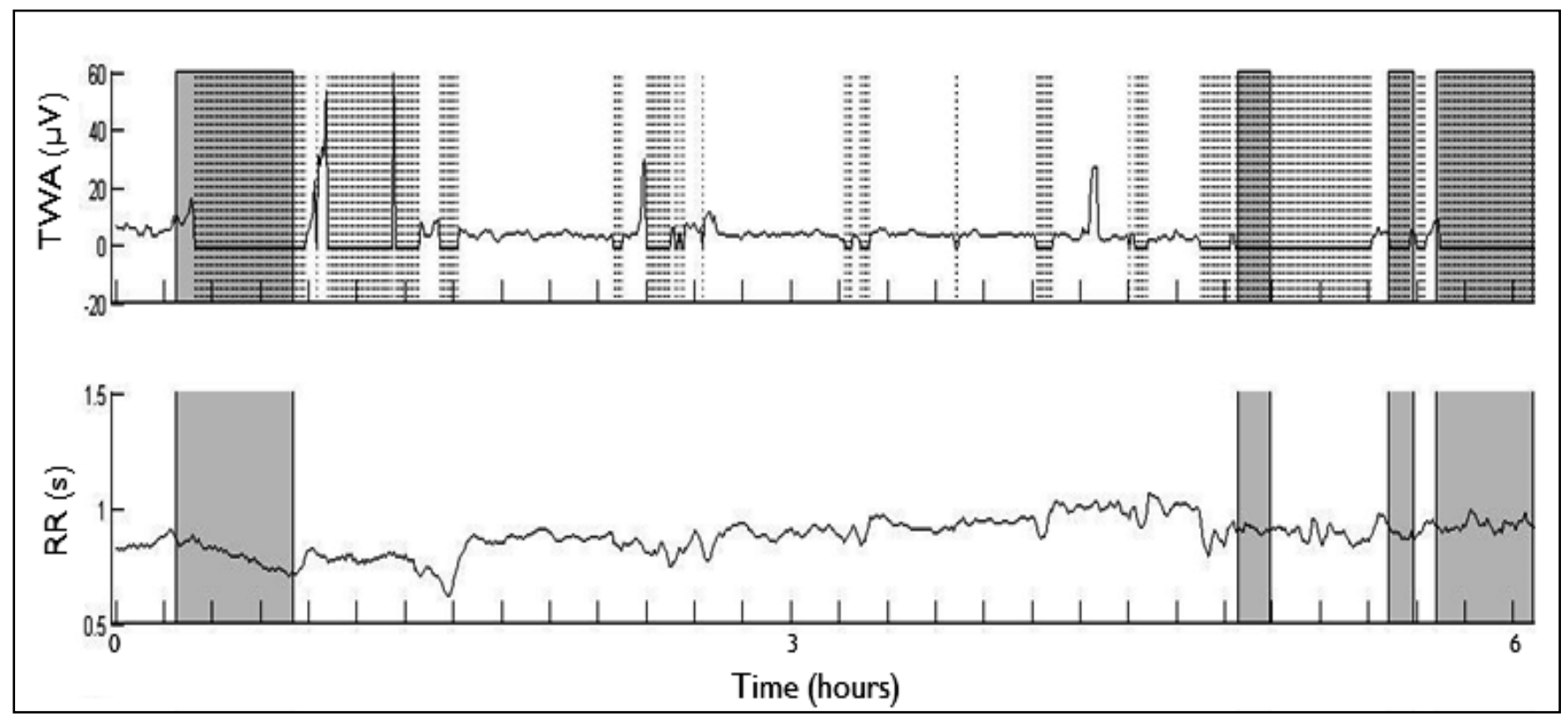

Figure 1. Overnight TWA and RR trends relative to patient number 14. Dotted lines are markers of not evaluable TWA (too high RRSD) whereas gray patches indicate occurrence of OA. 
to allow TWA identification (Table 1). Yet, TWA was identified in all patients. Results indicate that, overnight, average TWA is low but short, transient, higheramplitude TWA episodes usually occur. Moreover, they seem to occur in correspondence of regions characterized by higher HRV, often next to regions not suitable for TWA identification. Consequently, high-amplitude TWA episodes, and thus associated increment of cardiovascular risk, likely result underestimated. Future studies will evaluate the possibility of widening RRSD range for TWA identification in order to better assess highamplitude TWA episodes in SA patients.

\section{Conclusion}

Average overnight TWA is negligible, but short, transient, higher-amplitude episodes of TWA may occur during night indicating an increment of cardiovascular risk. Such episodes not necessarily occur during an SA episode; rather they are concomitant with an increased HRV so that they result difficult to be reliably identified.

\section{References}

[1] Floras JS. Sleep apnea and cardiovascular risk. Journal of Cardiology 2014;61:3-8.

[2] Somers VK, White DP, Amin R et al. Sleep apnea and cardiovascular disease. Circulation 2008;118:1080-111.

[3] Bradley TD, Floras JF. Obstructive sleep apnoea and its cardiovascular consequences. Lancet 2009;373:82-93.

[4] Kasai T, Floras JS, Bradley TD. Sleep apnea and cardiovascular disease: a bidirectional relationship. Circulation 2012;126:1495-510.

[5] Somers VK, White DP, Amin R et al. Sleep apnea and cardiovascular disease: an American Heart Association / American College of Cardiology Foundation scientific statement from the American Heart Association Council for High Blood Pressure Research Professional Education Committee, Council on Clinical Cardiology, Stroke Council, and Council on Cardiovascular Nursing. Circulation 2008;118:1080-111.

[6] Dorasamy P. Obstructive sleep apnea and cardiovascular risk. Therapeutics and Clinical Risk Management 2007;3:1105-11.

[7] Yamada S, Suzuki H, Kamioka M et al. Sleep-disordered breathing increases risk for fatal ventricular arrhythmias in patients with chronic heart failure. Circulation Journal 2013;77:1466-73.

[8] Lattimore JD, Celermajer DS, Wilcox I. Obstructive sleep apnea and cardiovascular disease. Journal of the American College of Cardiology 2003;41:1429-37

[9] Bloomfield DM, Hohnloser SH, Cohen RJ. Interpretation and Classification of Microvolt $\mathrm{T}$ Wave Alternans Tests. Journal of Cardiovascular Electrophysiology 2002;13:50212 .

[10] Burattini L, Zareba W, Burattini R. Assessment of physiological amplitude, duration, and magnitude of ECG T-wave alternans. Annals of Noninvasive Electrocardiology 2009;14:366-74.
[11] Burattini L, Bini S, Burattini R. Repolarization alternans heterogeneity in healthy subjects and acute myocardial infarction patients. Medical Engineering \& Physics 2012;34:305-12.

[12] Burattini L, Zareba W, Burattini R. Identification of gender-related normality regions for $\mathrm{T}$-wave alternans. Annals of Noninvasive Electrocardiology 2010;15:328-36.

[13] Man SC, De Winter PV, Maan AC et al. Predictive Power of T-wave Alternans and of Ventricular Gradient 2 Hysteresis for the Occurrence of Ventricular Arrhythmias in Primary Prevention ICD Patients. Journal of Electrocardiology 2011;44:453-9.

[14] Hohnloser S, Ikeda T, Bloomfield D et al. T-wave alternans negative coronary patients with low ejection and benefit from defibrillator implantation. Lancet 2003;362:125-6.

[15] Burattini L, Man S, Swenne CA. The power of exerciseinduced T-wave alternans to predict ventricular arrhythmias in patients with implanted cardiac defibrillator. Journal of Healthcare Engineering 2013;4:167-84.

[16] Ikeda T, Saito H, Tanno K et al. T-wave alternans as a predictor for sudden cardiac death after myocardial infarction. American Journal of Cardiology 2002;89:79-82.

[17] Burattini L, Man S, Fioretti S, Di Nardo F, Swenne CA. Heart-Rate Dependent Hysteresis of T-Wave Alternans in Primary Prevention ICD Patients. Annals of Noninvasive Electrocardiology 2016;21:460-9,

[18] Burattini L, Man S, Fioretti S, Di Nardo F, Swenne CA. Dependency of Exercise-Induced T-Wave Alternans Predictive Power for the Occurrence of Ventricular Arrhythmias from Heart Rate. Annals of Noninvasive Electrocardiology 2015;20:345-54.

[19] Nemati S, Malhotra A, Clifford GD. T-wave alternans patterns during sleep in healthy, cardiac disease, and sleep apnea patients. Journal of Electrocardiology 2011;44:126 2.

[20] Rechtschaffen A, Kales A. A Manual of standardized terminology, techniques, and scoring system for sleep stages of human subjects. Washington Public Health Service, US Government Printing Office, Washington DC, 1968.

[21] Chimaru Y, Moody GB. Development of the polysomnographic database on CD-ROM. Psychiatry and Clinical Neurosciences 1999;53:175-7

[22] Goldberger AL, Amaral LA, Glass L et al. PhysioBank, PhysioToolkit, and PhysioNet: components of a new research resource for complex physiologic signals. Circulation 2000;101:e215 - 20.

[23] Burattini L, Zareba W, Burattini R. Automatic detection of microvolt T-wave alternans in Holter recordings: effect of baseline wandering. Biomedical Signal Processing and Control 2006; 1:162-8.

[24] Burattini L, Zareba W, Burattini R. Adaptive match filter based method for time vs. amplitude characterization of microvolt ECG T-wave alternans. Annals of Biomedical Engineering 2008;36:1558-64.

Address for correspondence.

Laura Burattini

Università Politecnica delle Marche

Department of Information Engineering

Via Brecce Bianche,

63900 Ancona, Italy

1.burattini@univpm.it 